\title{
CLAVES TEÓRICAS DE LA PEDAGOGÍA SAPIENCIAL BAJOMEDIEVAL
}

\section{THEORETICAL KEYS OF THE SAPIENTIAL PEDAGOGY OF THE LATE MIDDLE AGES}

JAVIER VERGARA CIORDIA

UNED

LÍA VIGURIA GUERENDIÁIN

UNED - GEMYR

Recibido: 22/06/2021 Aceptado: 22/09/2021

\section{RESUMEN}

El presente estudio analiza cómo plantearon las gentes cultas de los siglos XII y XIII el ideal propio de la vida humana: vivir y ser feliz. Un desiderátum que, siguiendo la tradición, denominaron sabiduría y concretaron en la restauración de la vida humana, gracias a la actualización de la inteligencia y la voluntad, fortalecidas por la virtualidad de la gracia divina. Un proceso pedagógico complejo de síntesis, en el marco de una secularidad nueva, que ilustraron en numerosos trabajos y especialmente en cuatro obras clave de la pedagogía medieval: el Didascalicon de studio legendi, de Hugo de san Víctor (c. 1131); Disciplina scholarium, del Pseudo-Boecio (c. 1300), De eruditione filiorum nobilium, de Vicente de Beauvais (c. 1246) y De modo addiscendi, de Gilbert de Tournai (c. 1265). 
Palabras clave: sabiduría, inteligencia, voluntad, gracia sobrenatural, restauración pedagógica.

\section{ABSTRACT}

The present study analyzes how educated people of the twelfth and thirteenth centuries raised the ideal of human life: to live and be happy. A desideratum that, following tradition, they called wisdom and concretized in the restoration of human life, thanks to the updating of intelligence and will, strengthened by the driving force of spiritual grace. A complex pedagogical process of synthesis, within the framework of a new secularity, which they illustrated in several works and basically in four key works of medieval pedagogy: the Didascalicon de studio legendi, by Hugh of Saint Víctor (c. 1131); Discipline scholarium, from Pseudo-Boethius (c. 1300), De eruditione filiorum nobilium, by Vincent de Beauvais (c. 1246) and De modo addiscendi, by Gilbert de Tournai (c. 1265).

Keywords: wisdom, intelligence, will, supernatural grace, pedagogical restoration.

\section{HACIA UNA NUEVA SENSIBILIDAD}

1. Teniendo presente que los límites cronológicos en la periodización de la Historia son siempre artificiales, a la vez que metodológicamente necesarios, podemos decir, mutatis mutandis, que los siglos XII y XIII de la Baja Edad Media occidental constituyen un renacimiento cultural de primera magnitud. Un tiempo de tremendo calado y trascendencia que, mirando hacia afuera desde dentro, produjo una nueva sensibilidad caracterizada por profundas transformaciones psicológicas, políticas, religiosas, sociales y culturales, que elevaron la esperanza de las gentes e hicieron de esas centurias uno de los periodos más sugerentes y creativos del acontecer humano occidental ${ }^{1}$. Un tiempo nuevo que

1 El siglo XIII conforma con el siglo XII una unidad cultural que fue definida como el renacimiento cultural más importante de Occidente. Uno de los primeros investigadores que lanzó esta idea fue Charles Haskins cuando en 1927 publicó The Renaissance of the Twelfth Century. Obra clásica que ha perdurado hasta nuestros días y de la que se han hecho multiplicidad de ediciones y traducciones: Charles Haskins, El renacimiento del siglo XII. Barcelona: Ático de libros. 2013. Poco tiempo después aparecía publicado por Gérard Paré, Adrien Marie Brunet, Pierre Tremblay, and Gabriel Robert. 1933. "La Renaissance du XIIe siècle, les écoles et l'enseignement ". Paris: J. Vrin. Estudios más recientes han ahondado en el significado renacentista de esta época. Especialmente significativo es el estudio de Verger, J. La renaissance du XII siècle. Paris: CERF. 1996. Un estudio sobre los límites y alcance de este término puede verse en Soto Rábanos, J. Ma "Las escuelas urbanas y el renacimiento del siglo XII", en La enseñanza en la Edad 
sublimó el valor de la educación, convirtió la pedagogía en un valor cultural de primer orden y reivindicó la sabiduría como la meta más anhelada y sentida por las gentes cultas de la época.

2. Los escolásticos eran muy conscientes que el anhelo por la sabiduría no representaba una categoría nueva, se estaba ante un tema recurrente. Aristóteles ya sostenía que "todos los hombres por su propia naturaleza desean saber" (Metafisica, A1, 980 a, 22). Para la cultura veterotestamentaria se trataba del bien más preciado del hombre: "Vale más que las piedras preciosas, y cuánto hay de codiciable no puede comparársele" (Prov: 8, 11); y Cicerón, en De officis, concluía: estamos ante la primera de las virtudes, ante "la ciencia de las cosas divinas y humanas" (De officiis 1, XLVIII). Sentencias firmes que el alborear de la Baja Edad Media recuperó con empeño renovado. Hugo de san Víctor, siguiendo la tradición, recalcaba, en 1131: "el máximo consuelo en la vida es el afán por la sabiduría, el que la encuentra es feliz, y el que la posee es bienaventurado"'. Una reivindicación que presentaba un matiz innovador: no se trataba tanto de explicar el sentido de la naturaleza y de Dios, como el alborear lento de una nueva sensibilidad donde el hombre, con el apoyo simultáneo de la ciencia, la virtud y la gracia sobrenatural, aspiraba a construir su futuro a golpe de libertad.

3. El reto no era fácil, se trataba de alentar una nueva subjetividad. Los escolásticos, conscientes de ello, tenían a su favor el despertar de una secularidad diferente. El hombre culto del bajo medievo, sin renunciar a la fe, como valor supremo del hombre, y a la visión beatífica de Dios, como su fin último, empezó a considerar que vivir en el mundo y en sus realidades era lo óptimo y propio de la condición humana. Es el momento en el que se estabilizan los núcleos urbanos, en el que crece la población y la economía mercantil ${ }^{3}$; el tiempo en el que se redimensionan las artes mecánicas y las sociedades gremiales como cauce de socialización económica y profesional ${ }^{4}$; en el que se debilitan las

Media. X Semana de Estudios Medievales, coord. por José Ignacio de la Iglesia Duarte. (Logroño: Instituto de Estudios Riojanos. 2000): 207-242.

2 Hugo de san Víctor, Didascalicon de Studio Legendi (afán por el estudio). Introducción, estudio preliminar, traducción y notas de C. Muñoz Gamero Y M. ${ }^{a}$ L. Arribas. (Madrid: BAC-UNED, 2011), I, I, 6. A partir de ahora se citará: Didascalicon y la nomenclatura correspondiente a la obra.

3 La población europea a comienzos del siglo XI se estimaba en 73 millones, cuando en el siglo x era de 42 millones. De igual modo, la contratación monetaria, la especulación y los préstamos se convirtieron en hábitos y problemas cotidianos. Cfr. Jacques Le Goff, La Edad Media y el dinero. (Madrid: Akal, 2012).

4 Que la concepción de las artes mecánicas tuviera naturaleza estable en el marco de la cultura sapiencial no fue logro fácil. Hasta bien entrado el siglo XIII, los llamados saberes nobles se venían sustanciando en dos grandes áreas: la especulativa o teórica (scibilia) y la práctica o moral (agibilia). Las 
estructuras feudales para dar paso a la figura del Estado monárquico como forma indiscutida de gobierno 5 ; es la época en la que la recta regni administratio disminuye su querencia religiosa y moral para centrarse en aspectos más políticos, económicos y sociales de la res publica ${ }^{6}$; un momento en el que las relaciones entre naturaleza, medicina y educación se presentan como elementos determinantes de una nueva cultura que, junto con la dimensión individual, social y trascendente, debían ayudar al hombre a alcanzar la felicidad por la fuerza de la educación ${ }^{7}$.

artes mecánicas o saber productivo (factibilia) tenían el rango de saberes inferiores. Eran calificados con expresiones como inferiores, serviles, exteriores, etc. El porqué de esta devaluación obedecía a una concepción antropológica, heredada sobre todo de san Agustín, que entendía la condición humana en dos grandes dimensiones: el mundo interior y el exterior. El primero era el depositario de la verdad, el destinado a saborear la contemplación, la belleza, la felicidad; su instrumento regulador era las artes liberales, las destinadas a cultivar el espíritu. El mundo exterior, por el contrario, representaba lo contingente, lo material, lo caduco. A él correspondía la zona de la corporeidad material, regulada por las artes mecánicas, cuyo fin no era otro que satisfacer las necesidades de la vida terrestre (Agustín de Hipona, De Civitate Dei, XXII, 24.). Esta concepción llevó a considerar los saberes mecánicos menos dignos. Alberto Magno considerará que se trata de saberes que fuerzan el alma a desertar de ella misma, a exteriorizarse en lo sensible, por eso la materia se adultera, se desvirtúa (Alberto Magno, De natura et origine animae, I, 1). Tomás de Aquino los consideraba saberes innobles por realizarse sencillamente con las manos (Tomás de Aquino, Quaestiones de duodecim quodlibet, VII, 17, c. 5.).

5 Cfr. Carlos Estepa Díez, Instituciones y poderes en la Europa medieval. Imperios, reinos y principados. (Madrid: Síntesis, 2017).

6 El símbolo de este cambio, que suponía el fin del llamado agustinismo político y la emergencia del naturalismo social político, hay que ponerlo en 1159, cuando Juan de Salisbury publicó su Policraticus, sive de nugis curialum et vestigiis philosophorum, y su culminación en el De regimine principum ad regem Cypri, (ca. 1265), de Tomás de Aquino. Sobre esta cuestión consúltese: Juan de Salisbury, Policraticus. Editor Miguel Ángel Ladero Quesada et al., (Madrid: Editora Nacional, 1984). Juan de Salisbury, Policraticus o De las frivolidades de los cortesanos y de los vestigios de los filósofos. Traducción y notas, José Palacios Royán. Prólogo e introducción, Juan Gil y Gonzalo del Cerro. (Málaga: Universidad de Málaga, 2007).

7 La dimensión psicofísica del hombre descansaba en una larga tradición, que la caída del Imperio romano había dejado somnolienta, y que el renacer naturalista y médico de la Baja Edad Media revitalizó con fuerza. Tres vías contribuyeron a ello. Por un lado, la traducción de obras de la medicina greco-árabe: el Liber Pantegni, el Libro de la ciencia de Alfarabi (870-950), el Canon medicinae de Avicena (9801037) y el Liber ad Almansoren de Rhazes (860-932) fueron alguna de sus manifestaciones. En segundo lugar, la publicación y desarrollo de los regimina sanitatis o manuales de salud. Especialmente importantes fueron los trabajos del portugués Pedro Hispano (1205-1277), de Juan de Toledo (c.1195-c.1260), de Aldobrandino de Siena (1234-1256), de Arnau de Vilanova (1238-1311), de Bernad de Gordon (12601318), de Maino Mainieri (c.1295-1368), etc. Por último, cabe señalar las aportaciones de enciclopedistas no médicos como Guillermo de Conches y su Philosophia mundi (c.1130), Bartolomé de Glanville y su De proprietatibus rerum (1250), Tomás de Cantimpré y su De naturis rerum (1240), o Vicente de Beauvais y su Speculum doctrinale (1246). Obras que pusieron de manifiesto que la medicina no era sólo saber físico, ni una ciencia independiente, ni constituía un fin en sí mismo, sino una philosophia secunda que trascendía los límites de la pura ciencia médica para convertirse en una norma de vida orientada al bienestar del hombre que la pedagogía no podía dejar de lado. Cfr. Vern L. Bullough, Universities, medicine, and science in the medieval West. (Burlington, VT: Ashgate, 2004). 
4. Estamos, en definitiva, ante un atrezo y contexto muy distinto, con una implementación lenta, progresiva y desigual, que acarreó consecuencias muy variadas. Una de ellas, de extraordinaria significación, fue la aparición de centros oficiales del saber ${ }^{8}$. Se trataba de instituciones de muy diversa naturaleza. Las escuelas monacales renovadas ${ }^{9}$, las catedralicias, las conventuales ${ }^{10}$, palatinas, laicas, gremiales, urbanas ${ }^{11}$, universitarias, etc. fueron algunas de sus manifestaciones más patentes. Muchas surgieron al albur de las migraciones intelectuales alentadas por la búsqueda de ciencia y títulos académicos; otras tuvieron un marcado alineamiento profesional y laico; y muchas respondieron a necesidades culturales, religiosas y clericales de diversa índole. En cualquier caso, todas fueron un motor de dinamización, socialización y cambio, que, en distinta medida y proporción, hizo del saber una pasión practica irrenunciable, cuyo culmen y mejor ornato se denominó sabiduría ${ }^{12}$.

8 Su punto de partida hay que cifrarlo en la constitución Habita, proclamada por Federico Barbarroja, en 1155-58, para proteger jurídica y económicamente a los estudiantes. A finales del siglo XII, en 1191 y 1198 , el papa Celestino III consolidaba su práctica firmando dos bulas que garantizaban la posesión de los beneficios eclesiásticos a los clérigos que acudían a los centros en busca de ciencia y títulos académicos. Pero será en el siglo XIII cuando se asiente social y canónicamente esta costumbre con el canon XI del IV Concilio de Letrán (1215) y la decretal Super specula, de 1219 del papa Honorio III. Disposiciones que prescribieron que los prelados y cabildos enviasen durante cinco años personas hábiles a los centros del saber. Cfr. J. Verger, "La mobilité étudiante au Moyen Âge". Histoire de l'Éducation 50 (1991): 6590 .

9 A partir del siglo XI, la secularización creciente de la vida social y cultural, la amplitud de los estudios y la exigencia de los mismos llevó a tensionar la vida monástica por un aparente conflicto entre monaquismo y escolaridad. Dualidad que desembocó en diferentes reformas. Unas optaron por el lema: "la profesión del monje no es la ciencia, sino la oración" (monacus non doctoris, sed plangentis habet officium). Tesis que, llevada a su extremo, supuso el cierre de escuelas externas en muchos monasterios y el debilitamiento de la escuela interior. Cluny, por ejemplo, elevó la edad de ingreso a 20 años, y el Císter a 15. Otras tendencias, capitaneadas por reformadores de la talla de san Pedro Damiano y san Bernardo de Claraval, decidieron compaginar la vida ascética, monacal y religiosa con el cultivo profundo y serio de la Filosofia y las Sagradas Escrituras.

10 Especialmente importantes fueron las escuelas de los conventos de franciscanos y dominicos, que optaron por una pastoral más urbana, disciplinar e intelectual, marcando diferencias notables con las órdenes monásticas, más vinculadas a zonas rurales, y a una vida de clausura, piedad y recogimiento. Cfr. Bonifacio Palacios Martín, "Los dominicos y las órdenes mendicantes en el siglo XIII", en VI Semana de Estudios Medievales de Nájera. Coord. por José Ignacio de la Iglesia Duarte et al. (Logroño: Instituto de Estudios Riojanos, 1996): 29-42. A. García y García "Educación estamental: educación de monjes u órdenes mendicantes". Historia de la Educación en España y América. La educación en la Hispania Antigua y Medieval 1. Ed. B. Delgado, (Madrid: Ediciones Morata/Ediciones SM, 1992): 481-485.

11 La escuela urbana más común fue la de gramática. Eran muy variadas, podían ser dirigidas por los propios maestros teniendo un carácter privado; podían ser comunales, impulsadas por el municipio; y podían ser fruto de fundaciones de diversa índole que llevaban aparejadas tareas de enseñanza. Cfr. Susana Guijarro González, "Las escuelas de gramática en la Castilla bajomedieval (siglos XIII-XV)". Studia histórica. Historia medieval 36-2 (2018): 9-38.

12 La historiografía pedagógica se ha hecho eco abundante de este fenómeno. A título de síntesis se citan algunos títulos: VV.AA. Historia de la Universidad en Europa. Coord. W. Rüegg. Volumen I. Las 
5. Escudriñar su naturaleza, los elementos que la conforman, su sentido y alcance fue la gran pasión del hombre culto de la Baja Edad Media. Pero tanto o más que ello, le interesó su dimensión práctica. Se trataba de integrarla interiormente, de ser sabio para ser feliz. Un desiderátum que supuso la proliferación de numerosas publicaciones pedagógicas sobre cómo alcanzar la felicidad. Por su significación y alcance, sobresalen cuatro obras de notable trascendencia. En primer lugar, el Didascalicon de studio legendi, publicada hacia 1131 por el monje sajón Hugo de san Víctor. Se trata del sillar clave de la pedagogía medieval, del eje axial que sostuvo los pilares de la sabiduría escolástica y el crisol que alumbró sobremanera el modo de alcanzarla. "De entre todas aquellas cosas a las que debemos aspirar, _ decía Hugo - la primera es la sabiduría, en la que reside la regla del bien perfecto" ${ }^{13}$. De no menor significación es Disciplina scholarium, publicada a finales del siglo XII por un laico anónimo - conocido por el pseudo-Boecio - para enseñar la sabiduría a los estudiantes de la emergente Universidad de París ${ }^{14}$. En tercer lugar, debe citarse el De eruditione filiorum nobilium, publicada por el dominico Vicente de Beauvais, en 1246, obra que pasa por ser el primer tratado de pedagogía sistemática de la Baja Edad Media y donde la adquisición de la sabiduría constituye su objeto y razón de ser $^{15}$. Aunque el corolario de todo ello es Rudimentum doctrinae, publicada,

universidades en la Edad Media. Editado por Hilde de Ridder-Symoens. (Bilbao: Servicio de Publicaciones de la Universidad del País Vasco, 1995); P. Guilli, Former, enseigner, eduquer dans l'Occident medieval 1100-1450. (Paris : SEDES, 1999); J. Verger, Culture, enseignement et société en Occident aux XII et XIII siècles. (Rennes: Presses Universitaires de Rennes, 1999). Jean-Philippe Genet, La mutation de l'éducation et de la culture médiévales. Occident chrétien (XII siècle-milieu du XV siècle). (Paris: Editions Sele Arslan, 1999); VV.AA., La enseñanza en la Edad Media. X Semana de Estudios medievales. (Logroño, Instituto de Estudios Riojanos, 2000); J. Villa Prieto. "La enseñanza en la universidad medieval. Centros, métodos, lecturas". Tiempo y sociedad 26, (2017): 59-131, https://tiemposociedad.wordpress.com.

13 Didascalicon. I, I, 6.

14 Estamos ante un auténtico Jean-Philippe de la pedagogía medieval. De ello dan testimonio los 133 manuscritos que se conservan de la obra. En el siglo XV se editan más de 40 incunables. En el siglo XVI está la reimpresión de 1564 de Glareano, que aparece inserta en el tomo 64 de la Patrología latina de Migne, pero ya atribuida al Pseudo-Boecio. En la posteridad cabe destacar el trabajo de Edda Ducci, quien en 1967 publicó Un saggio de pedagogia medievale: il De disciplina scholarium dello Pseudo Boecio. Torino: Societa Editrice Internazionale. En 1976 Olga Weijers hizo una magnífica edición crítica: PseudoBoèce (Pseudo-Boethius). De disciplina scolarium. Edition critique, introduction et notes par Olga Weijers. (Leiden-Köln: Brill, 1976). Y en 1990, el profesor Antonio García Mesegosa hizo una traducción castellana que fue prologada magistralmente por el profesor Buenaventura Delgado. Antonio García Masegosa Pseudo-Boecio. Disciplina escolar (Barcelona: PPU, 1990).

15 Fue escrita por Vicente de Beauvais, hacia 1246, a petición de la Reina Margarita de Provenza, esposa de Luis IX de Francia (1214-1270), para que sirviera de guía educativa a los vástagos de la familia real capeta. La obra supone uno de los primeros tratados amplios de pedagogía sistemática elaborados en la Edad Media. Un tratado que reflexiona sobre el concepto de educación, sobre sus fines y su didáctica, sobre las distintas etapas de formación, sobre el currículo, los agentes personales, la educación social, la 
entre 1261 y 1268, por el franciscano Gilbert de Tournai. Se trata de la enciclopedia pedagógica por excelencia. Un tratado de cuatro partes - apenas estudiado por la historiografía pedagógica - que escudriñaba con minuciosidad la naturaleza sapiencial y el modo de actualizarla ${ }^{16}$.

\section{CONSECUENCIAS Y REALIDAD DE UN PECADO DE ESPECIE}

6. En todas estas obras y en otros escritos que podrían traerse a colación una idea común afloró con reiteración: la felicidad consiste en actualizar la vida humana conforme al ser del hombre, conforme a su naturaleza (vita viventibus esse). Esta tesis, de claro sabor aristotélico-tomista ${ }^{17}$, llevó a los escolásticos a un sólido análisis antropológico, donde dos de las facultades superiores del hombre: inteligencia y voluntad, ligadas a la virtualidad de la fe, cobraron especial protagonismo. Errar en su análisis y sistemática implicaba un error pedagógico proporcional al error antropológico. Hugo de san Víctor, consciente de la magnitud del tema, se ancló en el principio de autoridad grecolatino y patrístico especialmente agustiniano-, y, con seguridad, insistió: "Hemos comprobado que los antiguos dijeron muchas cosas acerca del ingenio y la virtud, pero no hasta el punto de que parezca que no ha quedado nada por decir" ${ }^{\prime 18}$. Faltaba una

educación femenina.... Temas de por sí diversos que, al ser tratados con amplitud y profundidad, permitieron sedimentar no sólo una buena parte de la teoría y práctica pedagógicas de la Baja Edad Media, sino marcar el camino a una parte muy importante de la pedagogía posterior. Vincentius Bellovacensis De eruditione filiorum nobilium. Estudio, traducción y notas I. Adeva, y J. Vergara Ciordia. Collectio scriptorum medievaelium et renascentium (Madrid, UNED/BAC, 2010). A partir de ahora se citará De eruditione..., más la nomenclatura de la obra.

16 La obra es uno de los intentos más ambiciosos de la cultura escolástica por abordar la sistemática de la pedagogía sapiencial. Una empresa que Tournai dividió en cuatro tratados, asignando a cada uno una misión causal. Al primero, le asignó la causa final. Un tratado de tres capítulos en los que describía el fin principal por el cual se ha creado el alma racional (propter sapientiam obtinendam), y el fin último al cual se ordena la sabiduría misma (la vida eterna). El segundo tratado es un análisis sobre la causa eficiente de la educación. A lo largo de siete capítulos, Tournai analiza gradualmente los agentes de la sabiduría. Como buen franciscano, que rezuma un fuerte agustiniano-aristotélico, nos dice con vehemencia que el principal agente de la educación es Dios mismo y por participación le siguen: los ángeles, Jesucristo, y los hombres elegidos. El tercer tratado es el De modo addiscendi. Se refiere a la causa formal de la educación. En sus seis partes y 68 capítulos, Tournai aborda la adquisición misma de la sabiduría con relación al maestro y al discípulo. Cierra la obra un cuarto tratado dedicado a la causa material de la educación y más en concreto a las ciencias que actualizan la sabiduría. Desgraciadamente este cuarto tratado, que Tournai consideraba "el más difícil y sutil", está perdido y sólo se conserva de él un rVesumen en el llamado códice de Florencia. Un estudio sobre esta enciclopedia véase en: Gilbert de Tournai De modo addiscendi (Sobre el modo de aprender). Edición bilingüe preparada por Javier Vergara Ciordia y Virgilio Rodríguez García Collectio sscriptorum mediavalium et renascentium (Madrid: UNED/BAC, 2014).

17 Tomás de Aquino, In IV Libros Sententiarum, d. 26, a. 3 sc 1.

18 Didascalicon, I, X, 18. 
conclusión muy importante por afirmar, cuyo honor sí cabe atribuir a la escolástica: sus artífices hicieron la síntesis más lograda y sistemática que hasta entonces se había hecho entre moral, ciencia y fe. Una síntesis que impregnó la cultura de sentido sapiencial convirtió al escolástico en un hermeneuta de la realidad total, y le invitó a dar respuesta a todas y cada una de las dudas e interrogantes del sentir de su época ${ }^{19}$.

7. Un desiderátum antropológico apasionante, que para los griegos emanaba del deseo innato de saber que confiere la naturaleza, y que para el cristianismo medieval surgía de una necesidad más profunda, sin la cual resultaba de todo punto imposible entender la cultura de la época: restaurar el orden y equilibrio de la naturaleza humana debilitada por el pecado original. (Gn 3: 1-24). Un drama mistérico y un daño inmenso al estado primitivo del hombre, calificado como un pecado de especie, que Tomás de Aquino resumió y describió con brillantez cuando en su Summa theologica afirmó: "Dios creó al hombre recto (Eccle 7: 30). En efecto, esta rectitud consistía en que la razón estaba sometida a Dios; las facultades inferiores a la razón; y el cuerpo, al alma. La primera sujeción era la causa de las otras dos, ya que, en cuanto que la razón permanecía sujeta a Dios, se le sometían a ella las facultades inferiores" ${ }^{20}$. Pero el pecado original rompió esa armonía, debilitó el alma y dejó una herida difícil de restañar, que el Aquinate definió como falta de orden para conseguir el fin ${ }^{21}$. Un orden que, de acuerdo con el Pseudo Hugo, en su De spiritu et anima, se restauraba con el descubrimiento de la verdad por la vía del entendimiento, se fortalecía con el bien por la práctica de la virtud, y se consolidaba con la ayuda de Dios, a través de la gracia santificante:

"El alma tiene en sí los principios de acción recibidos por su creador, por lo cual es perfecta en su género; consiguientemente desde que existe conocería todo lo que el hombre puede conocer, si no existiese la rémora o pesadez de la carne. Esto se puede probar por el primer hombre, desde el punto que existió, tuvo perfectamente la ciencia humana. Pero ahora, corrompida la humanidad, el alma desde que se une al cuerpo se corrompe y no puede ejercer su capacidad

19 Didascalicon, III, 7.

20 Tomás de Aquino, Summa Theologica, I, 95, 1 in c.

21 Tomás de Aquino, Summa Theologica, I-II, 85, 3 in c. "Esta justicia original desapareció por el pecado original, $\mathrm{y}$, como consecuencia lógica, todas esas fuerzas han quedado disgregadas, perdiendo su inclinación a la virtud. A esa falta de orden respecto del fin es a lo que llamamos herida de la naturaleza". 
hasta que, ejercida con el uso, la experiencia y la enseñanza de alguno, empieza a discernir la luz"22.

\section{MISIÓN DE LA INTELIGENCIA}

8. Implementar esta restauración, que reproduce fielmente las ideas agustinianas del De trinitate XIV, y del libro XXII de la Civitas dei, no resultaba tarea fácil. Se trataba de una vuelta al ser, de un retorno al estado ancestral del hombre, de un proceso pedagógico que los medievales tuvieron necesidad de explicar en todas y cada una de sus partes. Su punto de partida fue la inteligencia, facultad que consideraron el ornato más bello del alma, el atributo más parecido a la divinidad que tiene el hombre ${ }^{23}$. Una luz superior creada por Dios, impresa en la criatura humana en el momento de su creación, y participada por el hombre para captar las esencias de las cosas, trascender la materialidad de lo creado y acceder a la sabiduría, que es la meta donde reside la verdad y el bien perfecto ${ }^{24}$. Esta idea, de tremendo calado y trascendencia, formulada con brillantez por Juan de la Rochelle, Alberto Magno, san Buenaventura, Tomás de Aquino y otros escolásticos de pro, marcó sobremanera la gnoseología bajomedieval cristiana y puso al descubierto su signo de identidad más representativo: Dios, suprema y total verdad, era el principio y el fin de la inteligencia; fuera de él, el conocimiento era vano y todo carecía de sentido ${ }^{25}$.

9. Esta tesis, formulada con pasión a partir de finales del siglo XII, cuando se conoció en Occidente el De anima, la Metafisica y la lógica nova de Aristóteles, amén de la gnoseología árabe, sirvió de estímulo para que los escolásticos vertieran ríos de tinta sobre la naturaleza y modo de proceder de la inteligencia. Una preocupación de todo punto loable, que no se justificaba por sí misma sino en función de su objeto: el conocimiento de la verdad, el conocimiento de Dios. Propósito que, aunque se implementó por diferentes vías, vino marcado sobremanera por la teoría de la refracción pedagógica de impronta agustiniana y

22 Pseudo-Hugo, De Spiritu et Anima, PL, 40, XLVIII, 815. "Anima, inquit, a Creatore suo habet principia, ex quo est in genere suo perfecta; unde ex quo est, sciret omnia quae ab homine sciri possunt, nisi gravitas carnis esset. Quod per primum hominem, qui ante corruptionem humanitatis, ex quo fuit, perfecte scientiam habuit humanam, probari potest. Sed modo, corrupta humanitate, ex quo conjungitur corrumpitur, nec proprietates suas potest exercere, donec usu et experientia et alicuius doctrina excitata incipit discernere, veluti si quis cum subtili acie oculorum in tenebrosa detrudatur, videre tamen non potest ibi, nisi prius assuescat tenebris et lumen accendatur".

23 Gilbert de Tournai, De modo addiscendi, Pars III, cap. VII.

24 Boecio, De consolatione philosophiae, 3, 10, 1. 37.

25 Tomás de Aquino, Summa Theologica, I-II, q.3, a.8. 
tomista. Dios al crear la multiplicidad formal de la naturaleza y manifestarse en las Sagradas Escrituras ha reflejado en un gran espejo su voluntad y su ser para que el hombre, mediante el pensamiento, la meditación y la contemplación, pudiera disfrutar en la contingencia de la vida de la antesala de la felicidad eterna ${ }^{26}$.

10. La teoría de la refracción gnoseológica no era nueva, tenía una larga tradición. Ya en el Antiguo Testamento, el Libro de Daniel, al describir el Canto de los tres jóvenes, pide a todas las cosas creadas que bendigan y den gloria al Creador por ser un reflejo de su voluntad (Benedicite, omnia opera Domini, Domino... Dan 3: 5-12). De igual modo, fue San Pablo, en la Primera Carta a los corintios, quien de modo explícito formuló la pertinencia didáctica de la refracción indirecta al afirmar: "vemos ahora mediante un espejo, confusamente; entonces veremos cara a cara (1 Cor 13:12). Los neoplatónicos y los Padres de la Iglesia, especialmente san Agustín, insistirán en las virtualidades cognitivas de la refracción indirecta al hablar del speculum cogitationis y del speculum men$t i s^{27}$. En cualquier caso, se trata de un género literario que no llegará a constituirse como tal hasta la Baja Edad Media ${ }^{28}$. Con anterioridad, el término speculum aparecía aisladamente sin constituir un género propio. Habrá que esperar al dinamismo gnoseológico de las centurias bajomedievales para que la refracción adquiera carta de naturaleza estable, generando los libros-espejo más diversos ${ }^{29}$. Entre otros, podemos señalar: Speculum ecclesiae, de Honorius Augustodinensis, c. 1109; Speculum virginum, c.1127, de Conrado de Hirsau; Speculum universale, de Raoul Ardent, c. 1199; Speculum futurorum temporum, de Gébénon de Eberbach, c.1220; Speculum novitii, de Etienne de Sally, 1234, etc.

11. Aunque, sin duda, el símbolo más representativo de todos ellos será el Speculum maius (c. 1246) del dominico francés Vicente de Beauvais. En el capítulo VI de su prólogo — el Libellus apologeticus — se afirma: "Pienso que nadie estimará superflua o inútil la naturaleza misma de las cosas, que yo he descrito lo mejor que pude; nadie, digo, que, iluminado por la verdad misma, esté acostumbrado a leer en el propio libro de la creación, puesto ahí para que leamos el poder, la sabiduría y la bondad de Dios, creador, gobernador y conservador

26 Anónimo, J.-P. Migne (ed.), De modo dicendi et meditandi. Patrología latina 176 (Parisiis: apud editorem, in via dicta d'Amboise, 1845. En adelante $P L$ y número del volumen). La traducción y estudio crítico de esta obra puede verse en Revista Española de Pedagogía 238 (2007): 519-544.

27 San Agustín, De trinitate. XV, 20, 24, 44, 39. PL 42. Soliloquiorum libri duo, II, 6-10. PL 32.

28 Javier Vergara Ciordia, "Enciclopedismo especular en la Baja Edad Media: la teoría pedagógica del espejo medieval", Anuario de Historia de la Iglesia 18 (2009): 295-310.

29 Cfr. M. J. Muñoz Jiménez, P. Cañizares Ferris, C. Martín (eds.) La Compilación del saber en la Edad Media. (Turnhout: Brepols, 2013). 
de todas las cosas. Pues, como dice el gran Basilio, Aquellos que entienden la verdad y por las cosas visibles deducen las invisibles, aprecian la obra de nuestro benefactor en la tierra, en el agua, en el aire, en el cielo, y en todo lo que ven. Así, unido estrechamente el Señor a sus sentidos, ni se les da ocasión de pecar, ni se da lugar al enemigo para que sugiera cosas contrarias" ${ }^{\prime 30}$. Y en la misma línea, el franciscano Buenaventura de Bagnoregio, en su Itinerarium mentis in Deum (c.1257), recalcará: "El que con tantos esplendores de las cosas creadas no se ilustra está ciego; el que con tantos clamores no se despierta, está sordo; el que por todos estos afectos no alaba a Dios, ése está mudo; el que con tantos indicios no advierte el primer Principio, ése tal es un necio"31.

12. Esta apuesta, que impregnó de sentido secular y escatológico la cultura cristiana del medievo, ligando naturaleza, ciencia y Dios, tuvo dos consecuencias prácticas de considerable magnitud. Por un lado, la naturaleza ya no será una entelequia incausada y ordenadora del universo - como la llamaba Platón en el Timeo ${ }^{32}$-, ni un concepto difuso y de difícil comprensión, como lo llamaba Cicerón en De inventione ${ }^{33}$, será una creación divina, preñada de ilusión y esperanza, cuya radicalidad más genuina será reflejar de forma indirecta, en su multiplicidad y singularidad formal, la voluntad, el sentimiento y el ser de Dios, para hacer feliz al hombre y llevarlo a la eternidad ${ }^{34}$. "Aprendamos en la tierra la ciencia de aquello que perseverará con nosotros en el cielo", pues, como decía san Jerónimo, el saber sólo se desvanece en cuanto a la forma e imperfección, pero no en cuanto a su sustancia ${ }^{35}$. Por otro, esa diversidad formal, en tanto que reflejo indirecto de Dios, se presentaba como el contenido óptimo de la ciencia y del aprendizaje, era la gran posibilidad humana. Una dimensión práctica que Hugo de san Víctor supo resaltar cuando, con la contundencia acostumbrada, dijo: "En efecto, una cosa es no saber y otra muy diferente no querer saber. No saber es, sencillamente, propio de la debilidad, pero despreciar el

30 Vicente de Beauvais, "El Libellus apologeticus. Un símbolo del enciclopedismo medieval”. Estudio, traducción y notas por Javier Vergara. Educación XXI 6, (2003):149-202, (Cap. VI: Apologia de natura rerum et historia temporum: Porro ipsam rerum naturam, quam diligentius ut potui descripsi, nullus (ut aestimo) superfluam aut inutilem reputabit, qui in ipso creaturarum libro nobis ad legendum proposito, creatoris, gubernatoris et conservatoris omnium Dei potentiam, sapientiam et bonitatem, ipsa veritate rationem illuminante, legere consueverit.)

31 San Buenaventura, Itinerarium mentis in Deum (Madrid: BAC, 1945) V. I, 575.

32 Platón, Timeo, 35 ab.

33 Cicerón, De inventione. 1, 34.

34 Gilbert de Tournai, Rudimentum doctrinae, tractatus primus, Pars tertia: Tertia pars continet quoniam in via per sapientiam invenitur Deus in creatura, Cap. I, Quod finis cognitionis humanae est in creatura Deum invenire, fol. 46. Edit. Florentina.

35 Cfr. San Jerónimo, Epistolae LIII 9, PL 22. 
conocimiento es propio de una voluntad depravada" ${ }^{36}$. Un juicio inconmovible que llevará a los victorinos Hugo y Ricardo de san Víctor, junto con Vicente de Beauvais, a concluir: la restauración espiritual del hombre se operaba por la ciencia o doctrina: et quoniam ipsa restitutio sive restauratio per doctrinam efficitur, atque perficitur ${ }^{37}$.

13. Esta afirmación llevó a otra pregunta no menos importante: cuánta y qué ciencia aprender. Lo cierto es que los escolásticos eran optimistas por naturaleza y en su fuero interno dirían que toda la posible, pues al proceder las cosas de Dios y ordenarse a él, representaban una oportunidad única de conocer la verdad $^{38}$. Un problema arduo que afrontaron escribiendo grandes sumas del conocimiento, potenciando libros, bibliotecas y florilegios, y reivindicando el enciclopedismo como solución sapiencial y escatológica. La expresión más acabada de ese desiderátum fue el ya citado Speculum maius de Vicente de Beauvais. Un gran florilegio en él se muestra una clasificación cuatripartita del saber: sermonical, práctica, mecánica y teórica, que rompía con la tradicional división de la ciencia estoica, que hasta el siglo XII había dividido los saberes en física, lógica y ética, para brindar una nueva diversificación, de sesgo más inductivo, práctico y experimental. En el siguiente cuadro se muestra un resumen de los contenidos científicos de la época ${ }^{39}$ :

\begin{tabular}{|c|c|c|c|}
\hline ciencia & materia & libro & columnas \\
\hline \multirow{3}{*}{ sermonical } & uocabularium & (lib. I) & 80 \\
& grammatic & (lib. II) & 430 \\
& logica & (lib. III) & 90 \\
\hline \multirow{3}{*}{ practica } & monastica & (lib. IV-V) & 150 \\
& oeconomia & (lib. VI) & 74 \\
& politica & (lib. VII-X) & 438 \\
\hline \multirow{2}{*}{ mechanica } & lanificium & (lib. XI) & 8 \\
& armatura & (lib. XI) & 42 \\
\hline
\end{tabular}

36 Didascalicon, I, X, 18.

37 Vincentius Bellovacensis, Speculum doctrinale, lib. I, col. 2. Edición fotomecánica de la edición duacense de 1624. Graz-Austria, Akademische Druck-u. Verlagsanstalt, 1964. "et quoniam ipsa restitutio sive restauratio per doctrinam efficitur, atque perficitur". Esta idea se reflejó literalmente en el Didascalicon, VI, 14, de Hugo, y en el Liber excerptionum, I, I, 2 a 5, de Ricardo de San Víctor.

38 Santo Tomás, Summa theologica, I, q.45, a.2.

39 Cfr. S. LUSIGNAN, "Vincent de Beauvais: intentions et réceptions d'une oeuvre encyclopédique au Moyen Âge", en Actes du XIVe Colloque de l'Institut d'études médiévales, organisé conjointement par l'Atelier Vincent de Beauvais (A.R.Te.M., Université de Nancy II) et l'Institut d'études médiévales (Université de Montréal) 27-30 avril 1988. Cahiers d'Études Médiévals. Cahier Special, 4, (SaintLaurent: Bellarmin, Paris: J. Vrin, 1990), 103-104. 


\begin{tabular}{|l|c|c|c|}
\hline \multirow{7}{*}{} & ars theatrica & (lib. XI) & 5 \\
& nauigatio & (lib. XI) & 3 \\
& uenatio & (lib. XI) & 1 \\
& agricultura & $($ lib. XI) & 3 \\
& alchemia & $($ lib. XI) & 18 \\
& medecina practica & (lib. XI) & 95 \\
\hline \multirow{6}{*}{ theorica } & medecina theorica & (lib. XIII-XIV) & 296 \\
& physica & (lib. XV) & 134 \\
& mathematica & (lib. XVI) & 31 \\
& metaphisica & (lib. XVI) & 14 \\
& theologia & (lib. XVII & 43 \\
\hline
\end{tabular}

14. Esta síntesis puede decirse que refleja de forma aproximada los registros y modos que tenían los escolásticos de acercarse al conocimiento y unión con Dios. Una apuesta que pone al descubierto dos hechos de especial relevancia: por un lado, la historia de la ciencia es en buena parte la historia de la salvación humana, una circunstancia que impregnó el conocimiento medieval de un marcado acento providencialista y escatológico del que los escolásticos difícilmente pudieron sustraerse; por otro, esta dimensión condicionó sobremanera los criterios de verdad y los modos de validación del conocimiento, que se apoyó en siete grados de fiabilidad descendente: Sagradas Escrituras, decretos papales, cánones, legislación conciliar, escritos de los Padres de la Iglesia, escritores cristianos no canonizados, y autores paganos. Criterios referenciales que, a medida que se descendía en su escala, disminuía su certeza para dar entrada a una mayor discriminación y análisis crítico ${ }^{40}$. Praxis compleja que, en el marco de una secularidad creciente, dinámica y vital, demandaba con empeño la valentía de vivir cara a Dios con las exigencias del mundo, la libertad y la conciencia.

15. Un reto realmente apasionante que no estuvo exento de debates y divergencias metodológicas y gnoseológicas de calado. El siglo XII, por ejemplo, brindó la apuesta dialéctica de Abelardo, cuyos argumentos y modos de pensar dejaban en suspenso la afirmación de la verdad o lectio tradicional y ahondaban en la duda o quaestio como principio de argumentación. No menor relevancia alcanzaron los debates sobre la recién descubierta filosofía arábigo-aristotélica que, de la mano sobre todo de las paráfrasis de Avicena y de los comentarios de

40 El Libellus apologeticus, capítulo XI: De impari auctoritate eorum, quae excerpta sunt, col. 9, c, recoge una clasificación de autores y obras significativas dentro de cada nivel de certeza. También el capítulo XII: De ordine dignitatis earundem, col. 10, b, c, d. 
Averroes, proyectó en Occidente el corpus Aristotelicum, reivindicando el uso y ejercicio de un razonamiento más inductivo y experimental que, a la vez que abrían nuevos horizontes científicos, allegaba teorías imposibles de conciliar con la teología cristiana: la eternidad del mundo, de la materia, el eterno retorno, el determinismo de los astros, etc. fueron alguno de sus ejemplos De igual modo, el llamado nominalismo de Duns Escoto (1266-1308) y el fideísmo de Guillermo de Ockham (1285-1349) posibilitaron el inicio de una separación entre razón y fe, debilitaron la hegemonía de la lógica aristotélica y abrieron cauces a una voluntad más independiente que las centurias posteriores se encargaron de afirmar y amplificar.

\section{LA EDUCACIÓN MORAL}

16. El segundo de los ejes que sustancia la actualización de la sabiduría es el bien. Se trata del fin al que por naturaleza tienden todos los seres humanos, su principio dinámico fundamental. Una posesión que en su grado sumo se denomina felicidad y en su realización práctica virtud. Tesis de fuerte sabor aristotélico (Ética a Nicómaco I, I), que la practicidad funcional del estoicismo romano: Séneca, Cicerón, Marco Aurelio, etc., trasformó en un fin en sí mismo, y que la paideia paleocristiana redimensionó en una realidad medial que tenía en la caridad el principio y el fin de la felicidad. Este recorrido, reelaborado filosófica y teológicamente por los Padres de la Iglesia, especialmente por san Agustín y san Gregorio Magno, llegó a la Baja Edad Media bastante consolidado y maduro. Aun así, fue sometido a una profunda disección y análisis crítico. Trabajos como Scito te ipsum seu Ethica (1136), de Pedro Abelardo; Summa de vitiis et virtutibus, de Raoul Ardent (c.1192); Summa aurea (c.1229), de Guillermo de Auxerre; De bono (c. 1260), de Alberto Magno; Summa Theologiae (1274), de Tomás de Aquino, etc. ahondaron en su historicidad y naturaleza hasta el punto de que muy pocas cosas quedaron por decir. En cualquier caso, estos escritos coadyuvaron a conformar una definición precisa y sólida de virtus, que marcó la sensibilidad moral de la Baja Edad Media y de centurias posteriores.

17. Una sensibilidad que, con salvedades al margen, vino caracterizada por tres ideas marco. En primer lugar, los escolásticos tuvieron claro que el estado ideal del hombre en cuanto hombre es el estado de virtud, el estado de bien, que 
es lo más acabado y perfecto del ser ${ }^{41}$. Convicción firme que demandaba, por un lado, la sindéresis o capacidad de distinguir entre el bien y el mal, y, por otro, la idea clara y distinta de que el mal no tiene entidad por sí mismo, nada bueno o útil puede derivarse de él, no se relaciona con la perfección, implica defecto, debilidad o carencia; por tanto, no puede ser un fin ${ }^{42}$. En segundo lugar, fueron muy conscientes que el conocimiento de la felicidad o del ser no produce el acceso directo o posesión automática del bien, aunque lo presuponga y se ordene a ello ${ }^{43}$. El conocer es propio de la razón; en cambio, el bien es fruto de la acción, del obrar humano ${ }^{44}$, de la virtus. Una fuerza o disposición del alma, impresa en el hombre en el momento de su creación y orientada, por la virtualidad de los hábitos operativos buenos, a la perfección de sus potencias ${ }^{45}$, especialmente de la inteligencia y la voluntad, para predisponer sus afectos, emociones e impulsos a la práctica del bien ${ }^{46}$. Teleología que puso al descubierto una tercera conclusión: el fin de la educación es la virtud moral personalizada. Un matiz genuinamente paleocristiano, que los escolásticos amplificaron y entendieron como una disposición o camino medial de libertad y autodeterminación personal, alentada por una voluntad informada por la razón, que tiene su causa eficiente en la caridad y amor divinos y su causa final en una relación donativa, donde la optimización de la acción y la mejora intrínseca del otro constituye su plasmación más radical ${ }^{47}$.

18. Implementar esta praxis no resultaba tarea fácil, en ella se sustanciaba una parte central de la ansiada restauración humana. Reto que requería no sólo conocer la naturaleza de la virtud, sino saber cómo actualizarla. A este fin se orientaron numerosas obras pedagógicas. Se podrían citar, entre otras: el Didascalicon de studio legendi (c. 1131) del monje sajón Hugo de San Víctor; el De disciplina scholarium, del Pseudo-Boecio (c. 1201); la Rethorica Novissima (c.

41 Tomás de Aquino, "Non enim intendit natura solum generationem eius, sed traductionem et promotionem usque ad perfectum statum hominis, inquantum homo est, qui est virtutis status (In $I V$ Sententiarum. dist. 26, Q.1, a.1.). Véase también Summa Theologiae, Supplementum, q. 41, a.1 in c.

42 Tomás, Summa Theologica, I-II, q.55, a.3.

43 Tomás de Aquino, Summa Teologica I-II, q.56, a.3 in c "Los hábitos primeramente señalados (intelectuales) no se llaman absolutamente virtudes, porque no confieren el bien obrar, sino que dan facultad para ello". (Primi vero habitus non simpliciter dicuntur virtutes: quia non reddunt bonum opus nisi in quadam facultate).

44 Tomás de Aquino, Summa Theologica I-II, q.55, a.2.

45 Tomás de Aquino, "Virtus nominat quandam potentiae perfectionem". Summa Theológica I-II, q. 55 , a. 1 in c.

46 Tomás de Aquino, Summa Theologica, q. 25, a. 1.

47 Tomás de Aquino, "Est autem homo dominus suorum actuum per rationem et voluntatem: unde et liberum arbitrium esse dicitur facultas voluntatis et rationis. Illae ergo actiones propriae humanae dicuntur, quae ex voluntate deliberata procedunt". Summa Theologica I-II, q.1, a.1 in c. 
1235), de Boncompagno da Signa; el De eruditione filiorum nobilium, del dominico francés Vicente de Beauvais, (1247); el De modo addiscendi, del franciscano Gilbert de Tournai, (c.1263); el De modo docendi pueros, del dominico Guillaume de Tournai, (c.1264); Félix o libro de maravillas, (c. 1287), de Ramón Llull, etc. Una pequeña muestra, de un amplísimo elenco pedagógico, que tuvo como denominador común afirmar que la ratio, doctrina o ciencia sin virtud es vana; no lleva a la felicidad, requiere la educatio de las virtudes humanas ${ }^{48}$. Solo así se puede producir crecimiento, sólo así se da una auténtica eruditio o formación ${ }^{49}$. Término que en el pensamiento escolástico se sustanciaba en dos ideas clave: dominar el cuerpo con disciplina (educatio) y alimentar el entendimiento con ciencia o doctrina (instructio). Y todo ello - eruditio-, con un único fin: alcanzar la sabiduría por la práctica de la virtud. De ahí, que la pedagogía escolástica, retomando una máxima de Lactancio, concluya: la "virtud unida a la ciencia es la sabiduría" ${ }^{50}$.

19. En el plano moral, los escolásticos concretaron esta posibilidad en el cultivo asiduo y constante de las virtudes cardinales y dianoéticas por el carácter axial y dinámico de las mismas ${ }^{51}$, cifrando su realización práctica en la virtualidad de la disciplina ${ }^{52}$. Un concepto difuso y polisémico al que dieron una significación muy precisa: sujeción a norma ${ }^{53}$. Estamos ante la clave que trasforma el ejercicio en hábito virtuoso, en dominio intrínseco del espíritu. Un propósito que requería dos condiciones previas: confianza en el ejercicio reiterado de la acción y anuencia de la voluntad.

20. La primera exigencia, tutorizada de inicio por la guía magisterial o paterna, tiene como punto de partida los impulsos vitales primarios, busca liberar el espíritu de los lastres del pecado original e incorporar progresivamente la disciplina a la disposición natural, dejando el alma expectante, pronta para obrar y

48 Lactancio, Divinae institutiones, VI: De vero cultu 5. PL 6, 651A. "Sicut in itinere, inquit, celebrando nihil prodest viam nosse nisi conatus ac vires ambulandi suppetant, ita veri scientia nihil prodest, si virtutes propriae deficiant".

49 Vincentius Bellovacensis, De eruditione filiorum nobilium. Estudio, traducción y notas Adeva, I. y Vergara Ciordia, J. Collectio scriptorum mediaevalium et renascentium. (Madrid, UNED/BAC, 2010), Cap. 1,2 y $3,1-2$.

50 Lactancio, Divinae institutiones, III: De falsa sapientia, 8: PL 6, 369B. "Virtus, inquit, cum scientia coniuncta, est sapientia".

51 Pseudo-Boecio, De disciplina scholarium, PL. 64, 1234b.

52 De eruditione..., 25, 1. "se llama disciplina no sólo a la sujeción que se hace para la corrección, sino también a la misma corrección que sigue a la sujeción". (In duobus autem consistit puerorum eruditio, scilicet, ut a malo coerceantur et ad bonum informentur et utrumque pertinet ad disciplinam; nam disciplina dicitur et ipsa coertio quae fit ad correctionem et ipsa correctio quae sequitur coertionem).

53 De modo addiscendi, Pars II, cap. IX. 
limpia de vicios ${ }^{54}$. Ya Aristóteles había dejado claro que la parte irracional del alma precedía y condicionaba la parte superior, obnubilando la fuerza de la voluntad y del entendimiento (Politica, IV, 15; 1334b). De igual modo, san Agustín había sostenido que cuando el alma es incapaz de pensarse a sí misma, cuando la mente no es capaz de someterse a preceptos, se salva por la fuerza de la disciplina ${ }^{55}$. Por eso la razón no podía ser el principio de la virtud, sino el llamado por los estoicos natural indómito del alma. Un proceso de actualización complejo y largo, especialmente exigente y riguroso en la infancia y adolescencia por su debilidad moral, que se hacía autónomo a medida que emerge la razón práctica, y ésta, apoyada en la fuerza de la disciplina, se convierte progresivamente en norma rectora de conducta, dirigiendo los hábitos al ejercicio del bien.

21. La segunda de las exigencias, la anuencia de la voluntad, resultaba fundamental. Los escolásticos se resistían a reducir la disciplina a la resignación de una simple necesidad mecánica y determinista, derivada de la imperfección misma de la naturaleza humana. Se trataba de asumirla y aceptarla para trocar la necesidad en libérrima virtud. Para motivar esta idea, la escolástica recurrió, por un lado, a préstamos del estoicismo romano. Con Cicerón, dirá; "la práctica asidua dedicada a una sola cosa supera con frecuencia no sólo el ingenio, sino también la inspiración" 56 ; con Ovidio, insistirá: "nada hay mayor que la costumbre, acostúmbrate a lo que soportas mal; lo llevarás bien"57; y con Séneca —el más cristiano de los paganos-, concluirá: "el hombre bueno soportará con ánimo sereno cuanto le sucediere; pues sabrá que eso ha ocurrido por la ley divina, con la que todas las cosas funcionan"58.

22. Aunque el acicate más socorrido estará en el ejemplo de Cristo y los santos. En ellos se ha dado la suma perfección, un estado que han ganado por la aceptación voluntaria del ejercicio disciplinar. Cuando hay resignación hay solo quietud, no hay esperanza; cuando no se acepta la realidad no se es ético, se está fuera del camino, se está perdido (san Agustín, in Epistola Iohannis 10, 1,). Asumir la realidad y aceptarla es dinamismo, es el principio de la transformación y de la esperanza. Vicente de Beauvais dirá al respecto: quien sufre contra su total voluntad no tiene mérito ante Dios (1 Cor: 9, 24), y concluye: "si estáis fuera de la disciplina, de la cual todos han participado, es que sois bastardos y no legítimos" $" 59$ (Heb:12, 8:). Una aceptación que, cuando es asidua y constante, 
deriva en una metanoia progresiva, en una nueva índole o imagen virtuosa que lleva, de acuerdo con Cicerón, a una segunda naturaleza: Consuetudo quasi altera natura (De finibus $5,25,74$ ), a un nuevo ethos que por su misma entidad permite afirmar el espíritu y practicar la virtud ${ }^{60}$.

23. Un beneficio que los escolásticos cerraron con una matización clave: aunque la disciplina forme el carácter, la virtud no se tiene ni se posee en propiedad o plenitud al modo de una categoría material, simplemente se mantiene, pues es una cualidad intrínseca que siempre requiere y exige el uso de la libertad, para afrontar algo que en el plano práctico es inevitable: la imperfección. Con Séneca, los escolásticos recalcarán: "las imperfecciones tienen necesariamente que aparecer" ${ }^{61}$, forman parte del hombre, se nace con ellas y con ellas se muere; e incluso, cuando se superan y dominan por la virtud, aún debe mantenerse el uso de la disciplina, pues, como afirmaba Horacio, "aunque expulses el natural a golpes de horca, él volverá siempre". Lo único que cabe es domarlo, sujetarlo a norma y corregirlo para aspirar a que las caídas e imperfecciones de la vida sean ocasionales $\mathrm{y}$, si se puede, excepcionales ${ }^{62}$.

\section{LA VIRTUALIDAD DE LA FE}

24. El tercer elemento que sustanciaba el trípode de la felicidad es la fe. Una virtud a la que la cultura sapiencial de la Baja Edad Media rindió auténtica pleitesía. Los escolásticos tuvieron muy claro que el proceso de restauración humana no se agotaba en la virtualidad exclusiva del entendimiento y la virtus moral. Para ellos, resultaba de todo punto imposible pasar de un plano natural a otro sobrenatural por las solas fuerzas de la razón y la voluntad. Un planteamiento semejante implicaba limitar la naturaleza ontológica del hombre, y sobre todo olvidar el daño mistérico e insondable del pecado original. Agustín de Hipona ya sostenía que esa apuesta conllevaba una manifestación de soberbia, era un acto de idolatría y suponía una clara desobediencia. "El primero y mayor vicio para la perdición del hombre consiste en querer valerse de la propia y exclusiva autoridad"63. Para saciar su razón, para optimizar su voluntad, el hombre necesitaba del apoyo inconmensurable de la fe. Convicción firme que le llevó a asentar el aforismo: intelligo ut credam, credo ut intelligam, en un intento por

60 Gilbert de Tournai, Cfr. De modo addiscendi, Pars IV, cap. VI.

61 Séneca, Epistolae, 71, 35. Cfr. De eruditione, cap. XXIII.

62 Horacio, Epistolae, I, 10, 24. Cfr. De eruditione, cap. XXIII.

63 San Agustín, De Genesi ad Litteram libri duodecim, VIII, 6,12. Patrologia Latina 34 (Parisiis: apud editorem, in via dicta d'Amboise, 1845). 
afirmar que, aunque el entendimiento era la parte más noble del alma, por sí solo no podía desentrañar los misterios insondables de la naturaleza, necesitaba del fundamento de la fe, de la luz de la Revelación ${ }^{64}$. Este planteamiento, que impregnó la cultura medieval de un marcado tono fideísta, se mantuvo prácticamente incólume hasta las postrimerías altomedievales, momento en que fue revitalizado por la dialéctica de Anselmo de Canterbury (1034-1109), en dos de sus obras mayores: Monologion (cap. I) y Prosologion (II-IV), con el aforismo: Fides quarens intellectum, intellectus quarens fidem.

25. La escolástica, en el marco de una secularidad y diversidad creciente, no escudó el tema; es más, redimensionó la cuestión a unos niveles de disección crítica extraordinarios. Plano en el que, a la vez que reconocía las diferencias y la legítima autonomía de la razón y la fe, supuso un esfuerzo más que notable por defender su armonía y complementariedad. Numerosas obras alentaron esa exigencia. Entre otras: el De sacramentis christianae fidei, (c.1137), de Hugo de san Víctor; el Speculum fidei (c.1140) y Aenigma fidei (c.1144), de Guillaume de Saint Thierry; el Liber gratiae (c. 1244), de Vicente de Beauvais; el Breviloquium, (c.1257), de Buenaventura de Bagnoregio; la Summa contra Gentiles (c.1260) y la Summa theologica (1274) de Tomás de Aquino, etc. Una pequeña muestra de un amplio elenco, que tenía como base y referente fundamental la virtualidad del capítulo XI, de la Carta a los Hebreos: "la fe es la certeza de lo que se espera, la convicción de lo que no se ve" (Heb 11:1).

26. Estamos, quizás, ante uno de los versículos novotestamentarios de mayor influencia en la cultura escolástica. La clave es la palabra "certeza", equivalente al termino latino substantia y al griego hypostasis. (Est autem fides sperandarum substantia rerum, argumentum non apparentium). Concepto que para los escolásticos no expresaba tanto una convicción subjetiva como la manifestación, constancia y existencia cierta de una realidad vital en germen, que al crecer y actualizarse muestra la fuerza de Dios y el sentido de la vida eterna. Por tanto, la fe no era solamente un tender de la persona hacia lo que ha de venir, y que está todavía totalmente ausente; la fe nos da algo de la realidad esperada, y esta realidad presente constituye una "prueba" y "certeza" de lo que aún no se ve. Ésta atrae al futuro dentro del presente, de modo que el futuro ya no es el "todavía-no". El hecho de que este futuro exista, que sea real, cambia el presente y lo llena de esperanza; y así las realidades futuras repercuten en las presentes y las presentes en las futuras.

64 Cfr. San Agustín, Sermo 43, VII, 9. Patrología Latina 38 (Parisiis: apud editorem, in via dicta d'Amboise, 1845), col. 258. 
27. Esta realidad vital impregnó de esperanza la escolástica y la llenó de libertad al formular y despejar el camino de la sabiduría. San Buenaventura, que se recreó en todo ello, quintaesenció la cuestión afirmando con cierto laconismo: la fe es un don divino, una gracia que purifica, ilumina, vuelve perfecta el alma y la eleva a Dios adelantando en las contingencias de la vida terrena el sentido de la felicidad celestial. (Breviloquium, V, 1.2). Se trataba del valor más extraordinario del ser humano; algo incluso más valioso que el propio hombre con su dignidad personal y moral. "El bien de la gracia de uno - llegará a afirmar Tomás de Aquino- es mayor que el bien natural de todo el universo"65. Su virtualidad radicaba en elevar al hombre a la intimidad de Dios, en reintegrarlo a la condición de hijo adoptivo. Proceso que se iniciaba, consolidaba y crecía con la recepción de los sacramentos, cuyo efecto, según el Aquinate, era triple: hacer por la caridad divina que Dios habitara en el alma, elevar el entendimiento y la voluntad a la intimidad de Dios y añadir al alma la fuerza necesaria para alcanzar la perfección sin anular ni disminuir su propia libertad ${ }^{66}$.

28. Hasta aquí, la gran apuesta que la cultura bajomedieval brindó a la antropología de su tiempo. Parafraseando a Hugo de san Víctor, los escolásticos hicieron la síntesis más perfecta que hasta entonces se había hecho entre ciencia, moral y fe. Una tríada que al enraizarse en el interior del hombre otorgó unos aires de libertad autodeterminativa que hicieron de la Baja Edad Media uno de los periodos más sugerentes del acontecer humano occidental.

\section{REFERENCIAS BIBLIOGRÁFICAS}

Beauvais, Vicente de. De eruditione filiorum nobilium. I. Adeva, y J. Vergara Ciordia, estudio, traducción y notas. Collectio scriptorum medievaelium et renascentium. Madrid: UNED/BAC, 2010.

Beauvais, Vicente de. "Libellus Apologeticus: un símbolo del enciclopedismo medieval", Estudio, traducción y notas J. Vergara Ciordia. Educación XX1 6 (2003): 149-202.

Bullough, V. L. Universities, medicine, and science in the medieval West. Burlington, VT: Ashgate, 2004.

Cañizares Ferris, P. Coord. La Compilación del saber en la Edad Media. Turnhout: Brepols, 2014.

Ducci, Edda. Un saggio de pedagogia medievale. Il De disciplina scholarium dello Pseudo Boecio. Torino: Societa Editrice Internazionale, 1967.

65 Tomás de Aquino. Summa theologica, I-II, 113, 9 ad 2.

66 Tomás de Aquino. Summa theologica, I-II, 109-114. 
Estepa Díez, C. Instituciones y poderes en la Europa medieval. Imperios, reinos y principados. Madrid: Síntesis, 2017.

García Masegosa, Antonio. Pseudo-Boecio. Disciplina escolar. Barcelona: PPU, 1990.

García y García A. "Educación estamental: educación de monjes u órdenes mendicantes". En Historia de la Educación en España y América. La educación en la Hispania Antigua y Medieval. Vol. I, editado por Buenaventura Delgado Criado. Madrid: Ediciones Morata/Ediciones SM, 1992.

Genet, Jean-Philippe. La mutation de l'éducation et de la culture médiévales. Occident chrétien (XII siècle-milieu du XV siècle). Paris: Editions Sele. Arslan, 1999.

Guijarro González, Susana. "Las escuelas de gramática en la Castilla bajomedieval (siglos XIII-XV)". Studia historica. Historia medieval, 36-2 (2018): 9-38.

Gilbert de Tournai, Gilbert. De modo addiscendi (Sobre el modo de aprender). Edición bilingüe preparada por Javier Vergara Ciordia y Virgilio Rodríguez García. Collectio scriptorum media mediaevalium et renascentium. Madrid: UNED/BAC, 2014.

Guilli, P. Former, enseigner, éduquer dans l'Occident médiéval 1100-1450. Paris, SEDES, 1999, 2 vols.

Haskins, Charles. El renacimiento del siglo XII. Barcelona: Ático de libros, 2013. Hugo de San Víctor. Didascalicon de Studio Legendi (afán por el estudio). Introducción, estudio preliminar, traducción y notas de C. Muñoz Gamero y M. ${ }^{a}$ L. Arribas. Collectio scriptorum mediaevalium et renascentium. Madrid: BAC-UNED, 2011.

Le Goff, Jacques. La Edad Media y el dinero. Madrid: Akal, 2012.

Lousignan, S. "Vincent de Beauvais. Intentions et receptions d'une oeuvre encyclopédique au Moyen-Âge". Cahiers d'Études Médiévals. Cahier Special 4. Paris: J. Vrin, 1990.

Palacios Martín, Bonifacio. "Los dominicos y las órdenes mendicantes en el siglo XIII". En VI Semana de Estudios Medievales de Nájera. Coord. por José Ignacio de la Iglesia Duarte et al., Logroño: Instituto de Estudios Riojanos, 1996: 29-42

Paré, G., A. Brunet y P. Tremblay. La renaissance du XII siècle. Les écoles et l'enseignement. Ottawa: Publications de l'Institut d'Etudes Médiévales y Paris: Institut Catholique - Vrin, 1933

Pseudo-Boèce (Pseudo-Boethius). De disciplina scolarium. Edition critique, introduction et notes par Olga Weijers. Leiden-Köln: Brill, 1976.

Salisbury, Juan de y M. A. Ladero ed., Policraticus. Madrid: Editora Nacional, 1984.

Salisbury, Juan de. Policraticus o De las frivolidades de los cortesanos y de los vestigios de los filósofos. Traducido por J. Palacios Royán, introducción por J. Gil y G. del Cerro. Málaga: Universidad de Málaga, 2007. 
Soto Rábanos, J. Ma "Las escuelas urbanas y el renacimiento del siglo XII". En La enseñanza en la Edad Media. X Semana de Estudios Medievales, coordinado por José Ignacio de la Iglesia Duarte. Logroño: Instituto de Estudios Riojanos, 2000.

Verger, J. La renaissance du XII siècle. Paris: CERF, 1996.

Verger, J. "La mobilité étudiante au Moyen Âge". Histoire de l'Éducation 50 (1991): 6-90..

Verger, J. Culture, enseignement et société en Occident aux XII et XIII siècles. Rennes: Presses Universitaires de Rennes, 1999.

Verger, J. "Enciclopedismo especular en la Baja Edad Media: la teoría pedagógica del espejo medieval", Anuario de Historia de la Iglesia 18 (2009): 295310.

Villa Prieto, J. "La enseñanza en la universidad medieval. Centros, métodos, lecturas". Tiempo y sociedad 26, (2017): 59-131.

VV. AA. Historia de la Universidad en Europa. Coord W. Rüegg. Volumen I. Las universidades en la Edad Media. Editado por Hilde de Ridder-Symoens. Bilbao: Servicio de Publicaciones de la Universidad del País Vasco, 1995.

VV. AA. La enseñanza en la Edad Media. X Semana de Estudios medievales. Logroño, Instituto de Estudios Riojanos, 2000..

Javier Vergara Ciordia Dep. de Historia de la Educación y Educación Comparada

Facultad de Educación Universidad Nacional de Educación a Distancia - UNED

Calle Juan del Rosal, 14 28040 Madrid (España) https://orcid.org/0000-0002-6118-5905

Lía Viguria Guerendiáin GEMYR

Facultad de Educación

Universidad Nacional de Educación a Distancia - UNED

Calle Juan del Rosal, 14

28040 Madrid (España) https://orcid.org/0000-0001-6675-8656. 